\title{
IMPACTO DO EXERCÍCIO NA DOR CRÔNICA
}

IMPACT OF EXERCISE ON CHRONIC PAIN

IMPACTO DEL EJERCICIO SOBRE EL DOLOR CRÓNICO

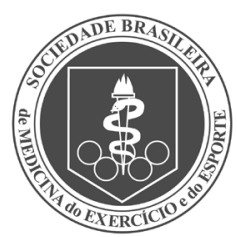

Artigo Original
Marlon Alves Subtil da Oliveira' (Médico)

Ricardo de Souza Campos

Fernandes' (Médico)

Samir Salin Daher (Médico)

1. Ambulatório de Medicina do Exercício e do Esporte do Hospital do Servidor Público Estadual (HSPE). 2. Instituto de Assistência Médica ao Servidor Público Estadual (IAMSPE) de São Paulo, São Paulo, SP, Brasil.

\section{Correspondência:}

Rua Mário Amaral, 459, ap.134,

Paraíso, São Paulo, SP,

CEP 04002-021

marlonsubtil@hotmail.com

\section{RESUMO}

Introdução: A dor é uma experiência subjetiva, que pode resultar de características teciduais, mas que também abrange fatores de várias ordens, incluindo os emocionais, culturais e individuais. A dor crônica é persistente ou recorrente, por mais de 3 meses, de causa desconhecida e não está necessariamente associada a uma lesão no organismo. Os gastos associados a essa condição de saúde estimulam o sistema a investigar intervenções eficazes para o tratamento das dores em geral. O fenômeno da analgesia induzida pelo exercício é sobretudo observado em atletas e os mecanismos envolvidos ainda são desconhecidos, embora haja tendência a aceitar a ação do sistema opióide endógeno. Objetivo: Investigar o efeito de um programa de exercícios físicos sobre a dor crônica inespecífica. Método: A amostra foi constituída por 29 indivíduos que aderiram a um programa de exercícios domiciliares. Sendo aplicado o Questionário Internacional de Atividade Física (IPAQ) que permite estimar o tempo semanal gasto em atividades físicas de intensidade leve, moderada e vigorosa e a Escala Visual Analógica (EVA) que foi utilizada para avaliar a intensidade da dor. A análise estatística foi realizada de forma descritiva e inferencial. Conclusão: A aplicação de programas multidisciplinares, predominando a prescrição de exercícios aeróbicos, de fortalecimento e alongamentos, em um protocolo de exercícios domiciliares apresentou melhora efetiva no limiar de dor em paciente com dor crônica inespecífica.

Palavras chave: dor crônica, exercício, medicina do esporte.

\begin{abstract}
Introduction: Pain is a subjective experience, which can result from tissue characteristics, but which also includes factors of various types, such as emotional, cultural and individual factors. Chronic pain is defined as persistent or recurrent pain, lasting for more than 3 months, of unknown cause, and not necessarily associated with a bodily injury. The costs associated with this health condition have prompted the system to investigate effective interventions for the treatment of pain in general. The phenomenon of exercise-induced analgesia is mainly observed in athletes, and the mechanisms involved are still unknown, although there is a tendency to attribute it to the action of the endogenous opioid system. Objective: To investigate the effect of a physical exercise program on nonspecific chronic pain. Method: The sample consisted of 29 individuals who joined a home exercise program. The International Physical Activity Questionnaire (IPAQ) was applied, which measures the weekly time spent in physical activity of light, moderate and vigorous intensity, and the Visual Analogue Scale (VAS), which was used to evaluate the intensity of the pain. The statistical analysis was performed using descriptive and inferential statistics. Conclusion: The implementation of multidisciplinary programs, with the prescription of aerobic exercises, and strengthening and stretching exercises, in a protocol of household exercises, presented an effective improvement in the pain threshold in patients with nonspecific chronic pain.
\end{abstract}

Keywords: chronic pain, exercise, sports medicine.

\section{RESUMEN}

Introducción: El dolor es una experiencia subjetiva, que puede resultar de las características de los tejidos, además de cubrir varios otros factores, como emocionales, culturales e individuales. El dolor crónico es persistente o recurrente, durante más de 3 meses tiene causa desconocida y no está necesariamente asociada con una lesión en el cuerpo. Los gastos asociados con este problema estimulan el sistema de salud para investigar intervenciones eficaces para el tratamiento de dolor en general. El fenómeno de la analgesia inducida por el ejercicio se observa especialmente en los atletas y los mecanismos involucrados aún se desconocen, aunque hay una tendencia a aceptar la acción del sistema opioide endógeno. Objetivo: Investigar el efecto de un programa de ejercicios en el dolor crónico inespecífico. Método: La muestra estuvo conformada por 29 individuos que se adhirieron a un programa de ejercicios en casa. Se utilizó el Cuestionario Internacional de Actividad Física (IPAQ), que permite estimar el tiempo semanal dedicado a actividad física de intensidad ligera, moderada o vigorosa, y la Escala Visual Analógica (EVA) para evaluar la intensidad del dolor. El análisis estadístico se realizó de forma descriptiva y por inferencia. Conclusión: La aplicación de programas multidisciplinarios, predominantemente la prescripción de ejercicios aeróbicos, de fortalecimiento y estiramiento, en protocolo de ejercicios en casa mostró una mejora efectiva en el umbral del dolor en pacientes con dolor crónica inespecífica.

Palabras clave: dolor crónico, ejercicio, medicina deportiva. 


\section{INTRODUÇÃO}

A dor é uma condição que todo ser humano procura evitar, independentemente de suas convicções, constituindo-se em experiência pessoal e subjetiva, resultado de características dos tecidos envolvidos em sua gênese, além de fatores emocionais, culturais e individuais. A dor constitui a interpretação do estímulo nocivo que protege o organismo e representa um verdadeiro sinal de alarme, sendo, paradoxalmente, um elemento vital ${ }^{1-5}$.

No contexto temporal, a dor pode ser classificada como aguda ou crônica. A dor aguda está associada à lesão do organismo, é de curta duração e, habitualmente, desaparece com a cicatrização desta lesão. Por outro lado, a dor crônica consiste em uma doença em si mesma, sendo reconhecida como tal pela 10ª Revisão do Código Internacional das Doenças (CID-10) da Organização Mundial de Saúde. A dor crônica é persistente ou recorrente e não está, necessariamente, associada à lesão orgânica. Sua causa nem sempre é evidente e pode, inclusive, ser indefinida. Consideram-se crônicas as dores em que o sintoma ou conjunto de sintomas mantém-se além do tempo fisiológico de cicatrização de determinada lesão, ou, então, as dores que permanecem por mais de três meses continuamente ${ }^{6-8}$.

Aproximadamente $80 \%$ das consultas médicas realizadas em todo o mundo deve-se à presença de dor ${ }^{9}$. Estima-se que 19\% da população brasileira sofra de dores crônicas. Nesta população predominam mulheres adultas jovens (média de idade de 37 anos), desempregadas e com nível socioeconômico baixo (10). Estudo recente mostrou que 75\% dos brasileiros que consultam serviços públicos de saúde relataram presença de dor crônica ${ }^{11}$.

O tratamento da dor é complexo e foram propostos programas multidisciplinares para combater a plasticidade do SNC formada pela persistência do sintoma álgico. Dentre as características dos programas multidisciplinares, predomina a prescrição de exercícios aeróbicos, de fortalecimento e alongamentos de músculos e tendões ${ }^{12,13}$.

O fenômeno da analgesia induzida pelo exercício é sobretudo observado em atletas. O primeiro a demonstrar esse fenômeno foi o médico Black, em 197914. Em seu experimento houve aumento no limiar de dor imediatamente após uma corrida de 40 minutos. Três décadas após esse primeiro estudo, os mecanismos envolvidos ainda não estão completamente esclarecidos, embora aceite-se a ação analgésica do sistema opióide endógeno ${ }^{14}$.

Castro et al., Cader et al., Caromano et al. e Lin et al. relataram que a utilização de exercícios isométricos, ativos livres e contrarresistidos, além do relaxamento de estruturas tensas ou contraturadas, foram capazes de reduzir edemas e processos inflamatórios, melhorando as condições circulatórias e favorecendo o alívio da dor e minimizando a incapacidade funcional. Os movimentos aplicados sob critérios personalizados podem auxiliar a dessensibilização de áreas dolorosas por meio da estimulação exteroceptiva, contanto que adequadas à capacidade de cada indivíduo ${ }^{5,15-17}$.

O objetivo deste estudo foi investigar o efeito de um programa de exercícios físicos sobre a dor crônica.

\section{MÉTODO}

Estudo aprovado pelo Comitê de Ética em Pesquisa do HSPE-IAMSPE, parecer NETIC 222/04 respeitando as normas estabelecidas na Resolução N 196/96 do Conselho Nacional de Saúde, de 10/10/1996, com relação à realização de pesquisa em seres humanos, sob protocolo $\mathrm{N}^{\circ}$ 0075/2008 UCB/VREPGPE/COMEP/PROCIMH.

Foi realizado um estudo prospectivo, no período de um ano, com pacientes do Ambulatório de Medicina do Exercício e do Esporte. Esses pacientes apresentaram dor por mais de três meses, aplicando-se um programa de exercícios domiciliares, compostos por exercícios resistidos, de alongamentos e aeróbicos ${ }^{12,13}$, com orientação de profissional de Educação Física. Foi realizada orientação inicial das atividades aos pacientes sedentários e restruturação das atividades dos que se declararam praticantes regulares.

Noventa e três pacientes foram pré-selecionados com dor crônica. Foram excluídos os pacientes que não efetuaram o retorno durante o prazo de coleta de dados. Desse modo, a amostra foi constituída por 29 indivíduos que aderiram ao programa. A maioria era do sexo masculino (55,2\%).

A média de idade foi de 51,7 $\pm 18,9$ (10 a 79 anos). Foram classificados como sedentários 12 (41,4\%) indivíduos e três (10,3\%) praticavam atividade física de forma irregular, 11 (37,9\%) praticavam atividade física regularmente, dois $(6,9 \%)$ praticavam atividade física de alto rendimento e um $(3,4 \%)$ praticava atividade física com outros objetivos.

Em relação ao diagnóstico principal da dor crônica, $9(31,0 \%)$ indivíduos foram diagnosticados com gonartrose 6 (20,7\%) pacientes com lombalgia (tabela 1).

O Questionário Internacional de Atividade Física (International Physical Activity Questionnaire - IPAQ) foi utilizado ${ }^{18}$. A versão curta do IPAQ, utilizada neste projeto, foi composta por sete questões abertas e suas informações permitiram estimar o tempo despendido, por semana, em diferentes dimensões de atividade física (caminhadas e esforços físicos de intensidades leve, moderada e vigorosa) e de inatividade física (posição sentada) ${ }^{18}$.

A Escala Visual Analógica (EVA) foi utilizada para avaliar a intensidade da dor e foi aplicada no início de cada atendimento, registrando-se o resultado numa escala de 0 a 10. ${ }^{19,20}$.

Foram incluídos neste projeto pacientes atendidos no Ambulatório de Medicina do Exercício e Esporte do HSPE-IAMSPE, com queixa de dor crônica que, após orientação adequada, apresentaram adesão ao programa de exercícios, prescrito segundo as capacidades físicas e fisiológicas de cada paciente, e que retornaram para reavaliação. Foi adotado como critério de exclusão qualquer contraindicação formal à realização de atividade física (estenose de canal medular, cardiopatia grave, deformidades, entre outras afecções), dor aguda e a não adesão ao programa de exercícios.

\section{Análise Estatística}

A análise estatística foi realizada de forma descritiva e inferencial. Os resultados foram apresentados por meio das medidas de posição (média), de dispersão (desvio padrão e amplitude) e distribuição de frequências

Tabela 1. Distribuição do diagnóstico principal na amostra estudada ( $n=29)$.

\begin{tabular}{c|c|c|c}
\hline Diagnóstico & N & Porcentagem & $\begin{array}{c}\text { Porcentagem } \\
\text { Acumulada }\end{array}$ \\
\hline Bursite & 3 & 10,3 & 10,3 \\
\hline Condropatia Patelar & 3 & 10,3 & 20,7 \\
\hline Dorsalgia & 1 & 3,4 & 24,1 \\
\hline Entesite & 1 & 3,4 & 27,6 \\
\hline Fasciíte Plantar & 2 & 6,9 & 34,5 \\
\hline Gonartrose & 9 & 31 & 65,5 \\
\hline Lombalgia & 6 & 20,7 & 86,2 \\
\hline Pubalgia & 1 & 3,4 & 93,1 \\
\hline Sacroileíte & 1 & 3,4 & 96,6 \\
\hline Sequela de Fratura & 1 & 3,4 & 100 \\
\hline Tendinite Calcâneo & 1 & 3,4 & \\
\hline
\end{tabular}


simples e relativa. O teste de correlação de Pearson foi utilizado para testar a relação entre o escore da EVA e a idade dos indivíduos. Para comparar o escore da EVA no início e no fim do estudo foi utilizado o teste $t$ de Student para dados pareados. Todas as análises foram realizadas utilizando o software Statiscal Package for Social Sciences para Windows (SPSS versão 18,0, Chicago IL, EUA) com o nível de significância de 5\%.

\section{RESULTADOS}

Nas informações referentes à intensidade da dor avaliada pela EVA verificou-se que na classificação predominou a intensidade moderada (4 a 7), em 21 (72,4\%) pacientes, seguido pela classificação intensa (oito a 10$)$ em sete $(24,1 \%)$ e leve (um a três) em um $(3,4 \%)$.

A média do escore da EVA no inicio do tratamento foi $6,7 \pm 1,7$, e de 2,0 \pm 0,6 após o tratamento. A média do escore da EVA no final do estudo foi significativamente menor que a média da EVA no início do estudo $\left(t_{28}=16,261 ; p<0,0001\right)$.

A tabela 2 apresenta a evolução da EVA de cada paciente de acordo com a idade, sexo e diagnóstico principal.

\section{DISCUSSÃO}

Este estudo sugere a eficácia de programa de exercícios no controle de processos álgicos, com diminuição significante do limiar de dor após a sua aplicação, corroborando com os achados de Castro et al ${ }^{5}$ de que programas de exercícios são efetivos em prevenir o aumento da dor, chegando inclusive a minimizá-la, resultando na aderência ao programa.

Neste estudo não foram considerados vários fatores de risco como tabagismo, obesidade, atividade laboral, estado psicológico, entre outros. Os indivíduos cumpriram pelo menos três meses do programa de exercícios e foram reavaliados em seu retorno.

Por ser um problema de saúde pública, a alta prevalência da dor crônica no Brasil apresenta impacto socioeconômico importante. Dados da Previdência Social em 2007 apontam que 20\% dos benefícios concedidos por afastamento do trabalho foram destinados a pacientes com dores crônicas ${ }^{21}$.

Os gastos associados a essa condição de saúde estimulam o sistema a investigar intervenções eficazes para o tratamento das dores em gera ${ }^{22}$. A atividade física bem orientada e acompanhada pode reduzir os gastos com medicação, além de reduzir o número de consultas e internações nesta classe de pacientes e pode significar um impacto econômico importante em tempos de dificuldades financeiras com a saúde em geral.

No que se refere ao diagnóstico principal, a maior parte dos indivíduos foram diagnosticados com gonartrose $(31,0 \%)$ e lombalgia $(20,7 \%)$ e representam doenças compatíveis com a média de idade de 51,7 anos encontrada no presente estudo. Assim como já havia sido verificado, essas doenças constituem influência dos altos níveis de inabilidade funcional e da maior fragilidade corporal, relacionando-se à perda da autonomia funcional ${ }^{23-26}$.

Houve uma melhor evolução nos pacientes com lombalgia, com redução média de 2,66 pontos na EVA, em comparação com os pacientes portadores de gonartrose, com apenas 0,88 pontos de redução média. Entretanto, ainda são necessários mais estudos para melhor avaliação dos resultados obtidos no presente estudo.

Existe a tendência a aceitar a ação do sistema opioide endógeno como mecanismo atuante no controle álgico da dor crônica por meio dos exercícios $^{16}$. O exercício físico, sobretudo o aeróbico, interage como modulador do aspecto desagradável da dor por intermédio do córtex, motivacional psicológico, do sistema nervoso autônomo (SNA) por meio da ação da dopamina e opioides liberados, dos mecanismos descendentes (noradrenalina, serotonina e peptídeos opioides) e da medula espinhal (opioides e $(G A B A)^{10}$. As respostas da ß-endorfina (opioide endógeno) ao exercício e ao treinamento já foram adequadamente demonstradas ${ }^{27}$.
Lin et al. evidenciaram que os ganhos no controle da dor crônica foram independentes do nível de comprometimento individual apresentado antes do início da intervenção realizada ${ }^{17}$.

Tabela 2. Evolução da EVA por paciente após tratamento conforme patologia, idade e sexo.

\begin{tabular}{|c|c|c|c|c|c|}
\hline $\begin{array}{l}\text { Idade } \\
\text { (anos) }\end{array}$ & Sexo & Diagnóstico & $\begin{array}{c}\text { Duração da } \\
\text { Queixa } \\
\text { (meses) }\end{array}$ & $\begin{array}{c}\text { EVA } \\
\text { (Primeira } \\
\text { Consulta) }\end{array}$ & $\begin{array}{c}\text { EVA } \\
\text { (Retorno após } \\
3 \text { meses) }\end{array}$ \\
\hline 44 & Masculino & Lombalgia & 6 & 5 & 3 \\
\hline 57 & Feminino & $\begin{array}{l}\text { Fasciíte } \\
\text { Plantar }\end{array}$ & 12 & 8 & 8 \\
\hline 65 & Feminino & Bursite & 6 & 5 & 3 \\
\hline 31 & Masculino & Pubalgia & 12 & 10 & 8 \\
\hline 20 & Masculino & $\begin{array}{c}\text { Condropatia } \\
\text { Patelar }\end{array}$ & 6 & 7 & 5 \\
\hline 65 & Feminino & Gonartrose & 12 & 8 & 7 \\
\hline 70 & Masculino & Gonartrose & 48 & 6 & 8 \\
\hline 79 & Feminino & $\begin{array}{l}\text { Lombocia- } \\
\text { talgia }\end{array}$ & 24 & 8 & 6 \\
\hline 56 & Feminino & Gonartrose & 12 & 3 & 5 \\
\hline 64 & Feminino & Gonartrose & 60 & 7 & 7 \\
\hline 34 & Masculino & $\begin{array}{l}\text { Fasciíte } \\
\text { Plantar }\end{array}$ & 6 & 8 & 2 \\
\hline 15 & Masculino & $\begin{array}{l}\text { Condropatia } \\
\text { Patelar }\end{array}$ & 12 & 7 & 1 \\
\hline 54 & Masculino & Lombalgia & 12 & 5 & 4 \\
\hline 38 & Masculino & Sacroileíte & 12 & 7 & 7 \\
\hline 46 & Feminino & $\begin{array}{c}\text { Condropatia } \\
\text { Patelar }\end{array}$ & 24 & 5 & 1 \\
\hline 22 & Masculino & Lombalgia & 6 & 5 & 1 \\
\hline 59 & Masculino & Bursite & 11 & 7 & 3 \\
\hline 61 & Feminino & Bursite & 24 & 6 & 3 \\
\hline 51 & Masculino & $\begin{array}{l}\text { Tendinite } \\
\text { Aquileana }\end{array}$ & 15 & 5 & 5 \\
\hline 53 & Masculino & Lombalgia & 4 & 6 & 2 \\
\hline 50 & Masculino & Gonartrose & 6 & 5 & 3 \\
\hline 64 & Feminino & Gonartrose & 12 & 10 & 8 \\
\hline 58 & Feminino & Gonartrose & 12 & 6 & 3 \\
\hline 10 & Masculino & Entesite & 12 & 5 & 4 \\
\hline 75 & Masculino & Dorsalgia & 7 & 8 & 6 \\
\hline 72 & Feminino & Gonartrose & 48 & 7 & 3 \\
\hline 73 & Feminino & $\begin{array}{c}\text { Sequela de } \\
\text { Fratura }\end{array}$ & 120 & 10 & 10 \\
\hline 74 & Feminino & Gonartrose & 60 & 9 & 9 \\
\hline 40 & Feminino & Lombalgia & 6 & 6 & 3 \\
\hline
\end{tabular}


A elaboração de programas educativos de prevenção e recorrência da dor pela atividade física deve ser direcionada no sentido de desencadear conhecimentos, atitudes e comportamentos compatíveis com uma dinâmica social fisicamente ativa e desenvolvida no quotidiano de vida, ao longo da existência das pessoas, independente da sua área de atuação.

\section{CONCLUSÃO}

A aplicação de um programa com predomínio da prescrição de exercícios aeróbicos de fortalecimento e alongamentos em um protocolo de exercícios domiciliares apresentou melhora efetiva no limiar de dor em paciente com dor crônica inespecífica, evidenciando que os exercícios são efetivos em prevenir o aumento da dor, chegando, inclusive, a minimizá-la, o que pode favorecer a aderência do paciente ao programa.

Todos os autores declararam não haver qualquer potencial conflito de interesses referente a este artigo.

\section{REFERÊNCIAS}

1. Ahlberg-Hulten G, Theorell T, Sigala F. Social support, job strain and musculoskeletal pain among female health care personnel. Scand J Work Environ Health. 1995;21:435-9.

2. Pimentel CAM. Dor crônica, terapia cognitiva comportamental e o enfermeiro. Rev Psiq Clín. 2001;28(6):288-94.

3. Budó MLD, Resta DG, Nicolini D, Büttenbender E, Pippi MC, Ressel LB. A cultura permeando os sentimentos e as reações frente à dor. Rev Esc Enferm. 2007;41(1):36-43.

4. Figueiró JA. A dor. São Paulo: Publifolha; 2000

5. Castro KVB, Silva ALS, Lima JMMP, Nunes WJ, Calomeni MR, Silva VF. Fisiomotricidade e limiares de dor: efeitos de um programa de exercícios na autonomia funcional de idosas osteoporóticas. Fisioter. Mov. 2010;23(1):161-72.

6. Bergqvist $\mathrm{V}$, Wolgast $\mathrm{E}$, Nilsson $\mathrm{B}$, Voos $\mathrm{M}$. The influence of VDT work on musculoskeletal disorders. Ergonomics. 1994;38:754-62

7. Bernard B, Sauter S, Fine L, Petersen M, Hales T. Job task and psychosocial risk factors for work-related musculoskeletal disorders among newspaper employees. Scand J Work Environ Health. 1994;20:417-26.

8. Bernard BP, editor. Work-related musculoskeletal disorders and psychosocial factors. Cincinati (OH): National Institute for Occupational Safety and Health; 1997.

9. Dickinson CE, Campion K, Foster AF, Newman SJ, O'Rourke MT, Thomas PG. Questionnaire development: an examination of the nordic musculoskeletal questionnaire. Appl Ergon. 1992;23:197-205.

10. Souza JB. Poderia a atividade física induzir analgesia em pacientes com dor crônica? Rev Bras Med Esporte, 2009;15(2), Mar/Abr.

11. Hildebrandt V. Back pain in the working population: prevalence rates in Dutch trades and professions. Ergonomics. 1995;38:1283-98

12. Lemstra M, Olszynski WP. The effectiveness of multidisciplinary rehabilitation in the treatment of fibromyalgia: A randomized controlled trial. Clin J Pain. 2005;21(2):166-74.

13. Souza JB, Bourgault P, Charest J, Marchand S. Long-term efficacy of the Interactional School of Fibromyalgia - a randomized controlled study. Arch Phys Med Rehabil. 2008; 85:1198-1204. In press 2008

14. Koltyn KF. Analgesia following exercise: a review. Sports Med. 2000;29(2):85-98.

15. Cader SA, Silva EB, Vale R, Bacelar S, Monteiro MD, Dantas E. Efeito do treino dos músculos inspiratórios so- bre a pressão inspiratória máxima e autonomia funcional de idosos asilados. Motricidade. 2007;3(1):279-88. 16. Caromano C, Ide MR, Kerbauy RR. Manutenção na prática de exercícios por idosos. Revista do Departamento de Psicologia - UFF. 200618(2):177-92.

17. Lin TY, Kaziyama HHS, Teixeira MJ. Síndrome dolorosa miofascial. In: Teixeira MJ, Figueiró JAB. Dor. São Paulo: Moreira Jr. 131-40, 2001.

18. Benedetti TRB, Antunes PC, Rodriguez-Añez CR, Mazo GZ, Petroskil EL. Reprodutibilidade e validade do questionário internacional de atividade física (IPAQ) em homens idosos. Rev Bras Med Esporte. 2007;13(1):11-6.

19. Clark P, Lavielle P. Martinez H. Learning from pain scales: patient perspective. J Rheumatol 2003;30(7):1584-8.

20. Ferraz MB et al. Reliability of pain scales in the assessment of literature patients with rheumatoid arthritis. J Rheumatol. 1990;17(8):1022-4.

21. Brasil. Regimento Interno do Instituto Nacional do Seguro Social - INSS. In: SISLEX: Sistema de Legis lação, Jurisprudência e Pareceres da Previdência e Assistência. DATAPREV, 2007. Portaria n. 026, de 19 de Janeiro de 2007. Dispõe sobre da natureza, sede e competência e da Estrutura Organizacional. Disponível em: <http://www010.dataprev.gov.br/sislex/paginas/66/MPS/2007/26.htm>. Acesso em: 20 dez. 2007.

22. Houtman I, Bongers P, Smulders P, Kompier M. Psychosocial stressors at work and musculoskeletal problems. Scand J Work Environ Health. 1994;20:139-45.

23. Andrade FA, Pereira LV, Sousa FAEF. Mensuração da dor no idoso: uma revisão. Rev Latino-am Enfermagem. 2007;14(2):37-45.

24. Andrade FA, Pereira LV, Souza FAEF. Mensuração da dor no idoso: uma revisão. Rev Latino-am. Enfermagem. 2006;14(2):271-6.

25. Dellarosa MSG, Pimenta CAM, Matsudo T. Prevalência e caracterização da dor crônica em idosos não institucionalizados. Cad Saúde Pública. 2007;23(5):1151-60.

26. Almeida HO, Versiani ER, Dias AR, Novaes MRCG, Trindade EMV. Adesão a tratamentos entre idosos Treatment adherence among the elderly. Com. Ciências Saúde. 2007;18(1):57-67.

27. Cunha GS, Ribeiro JL, Oliveira AR. Níveis de beta-endorfina em resposta ao exercício e no sobretreinamento. Arq Bras Endocrinol Metab. São Paulo. 2008;52(4):589. 